\title{
EDITORIAL
}

\section{"Racionalización del gasto en salud mental": Izquierda, izquierda, derecha, derecha, adelante, detrás, un, dos, tres...}

El poder del miedo y la yenka. La “yenka", la vieja y bastante "ratoneril" danza guatequera, ataca de nuevo. Y legiones de profesionales y técnicos la bailan y rebailan, tal vez felices porque, al menos, ellos pueden bailar.

Varias compañeras y compañeros, así como personas destacadas de nuestra asociación y nuestras profesiones y especialidades han recibido y recibirán en estos meses peticiones para colaborar en grupos de trabajo para redactar "Medidas de racionalización y ahorro" o "reducción del gasto en salud mental". Con todo mi respeto para los compañeros que adopten la postura de colaborar en los mismos, muchos de ellos incluso de nuestra misma asociación, y con mi comprensión hacia los que se sienten obligados a hacerlo para proteger a sus empleados --en el caso, cada vez más frecuente, de los servicios privados o semi-privados concertados--, creo que vale la pena dar algunas razones y argumentos disonantes con la postura más frecuente aceptación acrítica de la colaboración. Además, ahora que nuevamente se repite hasta la saciedad lo difícil que es distinguir la izquierda de la derecha en el momento actual, esta perspectiva divergente tal vez proporcione algún argumento coyuntural para esa diferenciación, además de los ya sabidos (y perfectamente válidos) que diferencian izquierda y derecha por la importancia concedida al internacionalismo, la ecología, la solidaridad como forma de organización y su consecuencia, la democracia real y cotidiana, la igualdad real ante la ley, la confianza en los hombres más que en su control, la equidad, la ayuda al desarrollo de los individuos y grupos más desfavorecidos, la denuncia de los medios y sistemas para el uso continuado de la mentira como arma de "destrucción masiva" de conciencias y voluntades, el pacifismo por contra de la "exportación de ejércitos" y la creación de "guerras locales", etc.

Los compañeros que nos hemos negado a participar con los administradores y políticos actuales en esos grupos de "racionalización del gasto" y otros que se muestran críticos con los mismos podemos aducir una serie de argumentos que tal vez ayuden a pensar un poco en nuestra situación concreta como país y en la situación de las disciplinas de la salud mental con respecto a la "casta parlamentaria":

1) Una cosa es la necesidad de participar en la organización y funcionamiento del sistema público de salud o de enseñanza en el cual trabajamos e incluso colaborar en la "reducción del daño" que, presumiblemente, los agentes de los especuladores transnacionales van a provocar en los sistemas sanitarios públicos europeos, y una actitud bien diferente es colaborar en grupos dirigidos, precisamente, a proporcionar un disfraz supuestamente técnico al desmantelamiento de esos sistemas públicos. No olvidemos 
que uno de los candidatos de los republicanos USA favoritos en las encuestas, Newt Gingrich, está ya defendiendo el trabajo de los niños mayores de 9 años de familias desfavorecidas, "combinada con su escolarización”. Indudablemente, es un marxista: Quiere reproducir lo que Karl Marx o Charles Dickens contaban en sus obras.

2) ¿Por qué se dice "racionalización” y no lisa y llanamente "reducción" o "ahorro"?: Edulcoración sintomática. ¿O es que esos nuevos gestores defienden en algún lado aumentar esos presupuestos para la salud mental?

3) Desde el punto de vista de la mayoría de la población, desde el punto de vista de la izquierda y la solidaridad, no hay ninguna demostración clínica, epidemiológica, económica o social que apoye el que haya que dedicar menores presupuestos a la salud mental en los años venideros, sino mayores. Orientando esos presupuestos de otra forma, como algunos llevamos diciendo en numerosas publicaciones y foros desde hace veinte años, pero con montos globales similares o mayores, no menores.

4) Lo cual plantea el tema de quién sufraga esos gastos. Algunos mantenemos la molesta opinión de que no debería ponerse en marcha ningún grupo de trabajo de reducción presupuestaria, si éste no comienza sus labores con una declaración que inste la fiscalía del estado a la persecución de los causantes de la crisis, a los causantes de los derroches y las "burbujas" (inmobiliarias, presupuestarias... y sanitarias). Tienen nombres, apellidos, NIF, empresas y entidades financieras. Habría que instar de forma imperativa a las autoridades públicas a no disminuir los gastos sociales, sino a hacer que los financien quienes especulativamente han creado esta crisis.

5) En el bien entendido de que también aquí izquierda y derecha se diferencian: Desde la izquierda real, la crisis se entiende como fundamentalmente social y política, y sólo secundariamente económica: Se dirime en ella quién tiene el verdadero poder en las democracias occidentales. Por eso la crisis y los aumentos del gasto social que va a implicar (en salud mental y en otros ámbitos) deben ser sufragados por los que han creado esta crisis. La población ha de saber que no colaboramos con ellos, sino que, si la situación se hace más grave, estamos dispuestos a asesorar en la reorientación de los fondos SIEMPRE QUE NUESTRAS AUTORIDADES PIDAN RESPONSABILIDAES PREVIAMENTE A LOS CAUSANTES DE ESTA SITUACIÓN y que cada grupo de trabajo lo pida en su declaración de intenciones: estamos hablando de la ruina de países enteros, de la ruina de sistemas sanitarios enteros, y eso supone centenares y miles de muertes e invalidaciones prematuras hoy evitables.

6) Como llevamos decenios defendiendo, los gastos en asistencia sanitaria, en general, deberían ser profundamente reorganizados. Pero no por la crisis, sino por la defensa y desarrollo de nuestro sistema sanitario, uno de los mejores del mundo a pesar de todo.

7) Hoy ya hay sobradas pruebas empíricas y experiencias a favor de una orientación de la atención a la psicopatología más basada en medidas psicológicas, psicoso- 
EDITORIAL

ciales y comunitarias, porque resulta menos derrochadora, disparatada, iatrogénica y, en definitiva, antisanitaria (y son términos que suenan a indignados, pero que escojo con cuidado y plena conciencia: "derrochadora, disparatada, iatrogénica y antisanitaria"). Una orientación del cuidado de la psicopatología basada en instituciones y terapias de un tipo diferente a las que hoy se llevan el grueso de los presupuestos de la red psiquiátrica: basada, en la realidad y no en los escritos, en la perspectiva comunitaria; basada, de forma mucho más amplia, en terapias psicológicas, psicosociales y biopsicosociales; basada en instituciones mucho menos costosas para el sistema y más eficientes, tales como los "acompañantes terapéuticos" adecuadamente cuidados y supervisados, residencias para crisis, equipos integrales de atención precoz, tratamientos integrales tipo Open Dialogue, Healing Homes, pisos asistidos, trabajos protegidos, actividades preventivas de la salud mental desde la infancia, "decrecimiento sostenible" del profesionalismo en la crianza y en las transiciones psicosociales y duelos, etc.

8) Por cierto, que, como han mostrado, entre otros, los economistas nórdicos --y cualquier persona interesada seriamente por la comunidad y la democracia--, esos son sistemas que poseen consecuencias tales como:

- Disminuir el paro (pues se necesita para ellos más trabajadores y profesionales).

- Aumentar las capacidades y el nivel de conocimientos de la población: Ese aumento de conocimientos en la población (y entre nuestros profesionales) es imprescindible para poner en marcha mediadas preventivas y anti-estigma, hoy ya suficientemente probadas aunque no financiadas. Y esa es otra vía para un "aumento del conocimiento" en la población general, uno de los escasos caminos que posee "la vieja Europa" para "salir" de esta crisis.

- Se trata de una vía concreta para reactivar la economía: desarrollar nuevos campos y agentes de creación, distribución e intercambio de bienes y servicios basados en una real extensión de los servicios comunitarios y personales, en una transición real desde nuestro "estado de medioestar" hoy en crisis hacia esa utopía que se ha llamado, muy exageradamente, "estado de bienestar".

9) Tales propuestas y datos, y miles de otros, sin embargo, no han sido tenidos en cuenta nivel administrativo y político, en especial en los últimos años. Y tanto por las autoridades políticas y administrativas entrantes como por parte de las, que, a menudo, han interpretado en este campo, torpe o ingenuamente, el papel del "aprendiz de brujo". $\mathrm{Y}$ ahora nos toca el aquelarre. Para que todo quedara bien claro, y sin posibilidades de confusión, posiblemente el primer servicio cerrado en Catalunya por parte del Servei Catalá de la Salut (que dice "organizar y controlar" la asistencia pública y la concertada) ha sido el primer equipo integral de atención a las psicosis, el EAPPP. El EAPPP era ya el único servicio comunitario de salud mental de Catalunya público, no concertado. Y ha sido cerrado a pesar de que ya había mostrado a nivel nacional e internacional su 
eficacia, eficiencia y efectividad. Y no sólo con datos clínicos, sino a nivel económico: reducción de hasta el $20 \%$ de los ingresos, reducción del coste de la medicación hasta la tercera parte para el mismo tipo de pacientes, reducción de los días de baja de los pacientes y sus familiares... Pero ha sido cerrado en septiembre del 2011 sin dar las más mínimas muestras de sensibilidad hacia el tema por parte de las autoridades sanitarias catalanas, sin oír siquiera a sus integrantes, tal vez en una manifestación más de su obediencia a otros intereses y/o pensamiento y/o perspectivas sanitarias (i), con lo que se convierten en "despedidoras a sueldo" de los especuladores transnacionales, lo sientan así o no.

¿Colaborar en la reducción presupuestaria con autoridades sanitarias, administrativas y políticas que, bajo diversas siglas, han mostrado la misma patente la falta de firmeza, criterio y credibilidad ante las presiones de los "burbujeadores" sanitarios? Hay que saber lo que se hace: Se trata de administradores que nunca han reclamado daños y perjuicios y ni tan siquiera han evaluado y denunciado conciertos y contratos ruinosos, hayan hecho lo que hayan hecho ciertos negociantes de la sanidad, algunos de ellos sumamente "piadosos". Esas autoridades sanitarias, ¿serán mejores que aquéllas para las que trabajamos más de diez años, consumiendo miles de horas de trabajo público como grupo de expertos, para desarrollar una guía de implementación de las terapias psicológicas en la red pública que ha sido postergada año tras año y que, probablemente, ya no será nunca aplicada? No parece una buena presentación por su parte, ni ofrece seguridad alguna de que nuestros trabajos no resulten baldíos o utilizados, como casi siempre, para alimentar el pensamiento sanitario más unidimensional.

En el momento presente, y en los años que vienen, parece que todos vamos a tener ocasión de tomar posturas en situaciones sumamente complejas. No es forzoso ver esa situación como ampliamente negativa, pues tal vez pueda despertarnos de un sueño más parecido a la embriaguez que al soñar, y que ha sido derrochadoramente alimentado con ríos de psicofármacos. No va a quedar más remedio que despertar de nuevo a la política. Cada uno tendrá que escoger a qué política. Porque todos aquellos "mantras" de que la izquierda y la derecha no se diferencian, todo es lo mismo, la izquierda ha muerto, la izquierda no tienen alternativas, la TINA omnipresente ("there is not other alternative"), tales "mantras" han revelado ya, incluso en nuestro ámbito, su carácter de "jaculatoria auto-consoladora". Y detrás, o enfrentamos con indignación y autogestión el poder del miedo que nos tenía atenazados, o nuevamente nos vestimos con "racionalidades" tan elementales y pobres que pronto tendrán razón para gritarnos...”¡Pero si van desnudos!". 